\title{
Accuracy of IgM antibody testing, FQ-PCR and culture in laboratory diagnosis of acute infection by Mycoplasma pneumoniae in adults and adolescents with community-acquired pneumonia
}

Jiuxin $\mathrm{Qu}^{1}, \mathrm{Li} \mathrm{Gu}^{1}$, Jiang Wu², Jianping Dong ${ }^{3}$, Zenghui $\mathrm{Pu}^{4}$, Yan $\mathrm{GaO}^{5}$, Ming Hu $\mathrm{H}^{6}$, Yongxiang Zhang ${ }^{7}$, Feng $\mathrm{GaO}^{8}$, Bin $\mathrm{Cao}^{1 *}$, Chen Wang ${ }^{9}$, for Beijing Network for Adult Community-Acquired Pneumonia (BNACAP)

\begin{abstract}
Background: Diagnosis of community-acquired pneumonia (CAP) caused by Mycoplasma pneumoniae in adults and adolescents is hampered by a lack of rapid and standardized tests for detection.

Methods: CAP patients from 12 teaching hospitals were prospectively and consecutively recruited. Basic and clinical information, throat swabs and paired sera were collected. Mycoplasma pneumoniae was detected by lgG and IgM antibody tests, fluorescence quantitative polymerase chain reaction (FQ-PCR) and culture. A comparative study of the diagnostic values of three methods, including sensitivity, specificity, positive and negative predictive values and positive likelihood ratio (PLR) was conducted. A fourfold or greater increase of IgG antibody titers of paired sera was set as the diagnostic "gold standard".
\end{abstract}

Results: One hundred and twenty-five CAP patients (52.8\% males, median age 47 years, range 14-85) were enrolled. Twenty-seven (21.6\%) patients were diagnosed with acute Mycoplasma pneumoniae infections by the "gold standard". Specificity values of all three methods were around 90\%. An increasing trend of sensitivity, positive predictive value and PLR was found, with the lowest in IgM testing (7.4\%, 28.6\% and 1.45), intermediate in FQ-PCR (40.7\%, 50\% and 3.63), and highest in culture $(55.6 \%, 75 \%$ and 10.9$)$.

Conclusions: In the defined group of patients, there was a good agreement between positive rate of MP cultivation of throat swabs and acute M. pneumoniae infection (PLR of 10.9). Since the sensitivity is low in all of the evaluated methods, the logical approach would be to incorporate PCR, culture and serological tests for optimum diagnosis of acute Mycoplasma pneumoniae infections in adults and adolescents.

Keywords: Community-acquired pneumonia, Mycoplasma pneumoniae, Diagnosis, Adult

\footnotetext{
* Correspondence: caobin1999@gmail.com

'Department of Infectious Diseases and Clinical Microbiology, Beijing Chao-

Yang Hospital, Capital Medical University, Beijing Institute of Respiratory

Medicine, Beijing 100020, China

Full list of author information is available at the end of the article
} 


\section{Background}

Mycoplasma pneumoniae (M. pneumoniae, MP) is a common human respiratory tract pathogen that causes 6-30\% of community-acquired pneumonia (CAP) cases in adults all over the world [1], and especially in China, where the incidence is $20-30 \%$ [2,3]. Since the clinical and laboratory manifestations do not differentiate between pneumonia caused by typical or atypical pathogens [4,5], and the commonly described $\beta$-lactams are not effective because M. pneumoniae lacks a cell wall, adequate laboratory diagnosis of $M$. pneumoniae pneumonia is important.

Diagnosis of $M$. pneumoniae infection in routine clinical practice has been based on serology, culture and polymerase chain reaction (PCR). Among these, paired sera showing a fourfold increase in IgG antibody titer has been considered as a more reliable method for the diagnosis of current $M$. pneumoniae infection [6-8]. However, because of difficulty in obtaining convalescent serum and time constraints, IgG antibody titer tests of paired sera are an epidemiological rather than a diagnostic tool. Culture of this organism is slow [9], and the correlation between culture and infection is tenuous because of the asymptomatic infection caused by $M$. pneumoniae $[10,11]$. The detection of IgM antibody titer in acute stage serum and fluorescence quantitative PCR (FQ-PCR) of throat swabs are faster techniques, but until now, a direct comparison between paired sera results and the above three assays, especially the FQ-PCR kit approved by State Food and Drug Administration of China, has not been made.

This study is based on the Beijing Network for Adult Community-Acquired Pneumonia, which consists of 12 teaching hospitals in Beijing. We evaluated the accuracy of IgM serology, FQ-PCR and culture for early diagnosis of CAP caused by $M$. pneumoniae, with a fourfold or greater increase of IgG antibody titers of paired sera as the "gold standard".

\section{Methods}

\section{Study population}

A prospective study was conducted in 12 teaching hospitals (Beijing Chao-Yang Hospital, Beijing Haidian Hospital, YanTai Yu Huangding Hospital, Peking University People's Hospital, Luhe Teaching Hospital of the Capital Medical University, WangJing Hospital of China Academy of Chinese Medical Sciences, Peking Union Medical College Hospital, China-Japan Friendship Hospital, Beijing Friendship Hospital, Beijing Pinggu Hospital, Huairou the First Hospital and Air Force General Hospital, PLA) in Beijing, China. From October 2010 to September 2011, adults and adolescent patients $(\geq 14$ years of age) with radiographically confirmed CAP were enrolled consecutively. M. pneumoniae was detected by the central laboratory, Clinical Microbiological Laboratory of Beijing Chao-Yang Hospital. Patients with immunosuppression and those who had received immunosuppressive therapy were excluded. In addition, pregnant or lactating mothers, patients from nursing homes, patients whose onset time was longer than 7 days or patients who had been admitted to a hospital longer than 2 days within the last 90 days were also excluded. Clinical features, including comorbidities (such as diabetes, heart diseases, cerebral vascular disease, chronic lung and renal disease) and laboratory data, were recorded on a data sheet and then entered into a computer database when patients were enrolled. The study was approved by the institutional review board in Beijing Chao-Yang Hospital. Written informed consent was provided by all adults and the parents of patients aged less than 18 years.

\section{Microbiological laboratory tests}

Throat swabs and first serum samples were obtained on admission, and convalescent serum samples were obtained 2-4 weeks later. Throat swabs were performed with $2 \mathrm{ml}$ transport broth medium (CM403, OXOID). Specimens were stored at $-80^{\circ} \mathrm{C}$ until transportation on ice to the laboratory within 2 weeks.

For $M$. pneumoniae culture, throat swab specimens were vortexed, supplemented with amphotericin B and penicillin, and inoculated into SP-4 medium. The medium was incubated at $37^{\circ} \mathrm{C}$, and observed daily for 2-6 weeks for a decrease in $\mathrm{pH}$ (a red to yellow color change). Positive cultures were confirmed by PCR assay as previously reported [12].

DNA was extracted from $200 \mu$ of throat swab sample by manual nucleic acid extraction (Qiagen QIAmp DNA Mini Kit, Valencia, CA). M. pneumoniae was detected by a commercial FQ-PCR kit (Daan Gene, Guangzhou, China) approved by State Food and Drug Administration, targeting the $16 \mathrm{~S}$ ribosomal RNA gene [GenBank:AF132740]. The FQ-PCR mixture was prepared in a total volume of $45 \mu \mathrm{l}$, containing $3 \mu \mathrm{l}$ of sample DNA. FQ-PCR was performed under the following conditions: initial activation at $93^{\circ} \mathrm{C}$ for $2 \mathrm{~min}$, followed by 10 cycles at $93^{\circ} \mathrm{C}$ for $45 \mathrm{~s}$ and $55^{\circ} \mathrm{C}$ for $1 \mathrm{~min}$, and 30 cycles at $93^{\circ} \mathrm{C}$ for $30 \mathrm{~s}$ and $45^{\circ} \mathrm{C}$ for $30 \mathrm{~s}$. The results were displayed as qualitative. An internal control, targeted human ribonuclease protein (hRNP) gene, was incorporated. The amplifications were performed using the AB 7500 Real Time PCR System (Applied Biosystems, Foster City, CA) according to the manufacturer's instructions.

The specimens were quantitatively tested for IgG and IgM antibodies against M. pneumoniae using the Virion/Serion ELISA kit (GmbH Germany ESR127G and ESR127M). Antibody activities were given in $\mathrm{U} / \mathrm{ml}$. Based on the manufacturer's recommendation, MP IgG calculation was interpreted as follows: positive results (> $30 \mathrm{U} / \mathrm{ml}$ ), borderline results $(20-30 \mathrm{U} / \mathrm{ml}$ ) and negative results $(<20 \mathrm{U} / \mathrm{ml})$; and for IgM: positive results 
(> $17 \mathrm{U} / \mathrm{ml}$ ), borderline results $(13-17 \mathrm{U} / \mathrm{ml}$ ), and negative results $(<13 \mathrm{U} / \mathrm{ml})$. All ELISA reactions were performed according to the manufacturer's instructions on the Thermo Multiskan MK3 (Thermo Fisher Scientific, Waltham, MA) system by the same technicians.

Microbiological examination was also performed in throat swab, sputum, urine and blood. The etiology was considered definite if one of the following criteria was met: (1) positive bacterial culture; (2) positive urinary antigen for L pneumophila (Binax Now L pneumophila urinary antigen test; Trinity Biotech, Bray, Ireland); (3) positive urinary antigen for $S$ pneumoniae (Binax Now $S$ pneumonia urinary antigen test; Emergo Europe, The Netherlands); (4) detection of respiratory viruses by RT-PCR using a Seeplex RV Detection Kit (Seegene Biotechnology Inc., Seoul, Korea), including respiratory syncytial virus $\mathrm{A}$ and $\mathrm{B}$, influenza virus (IFV) A and B, parainfluenza virus (PIV) 1-4, human rhinovirus (HRV), enterovirus, human coronavirus (229E/NL63 and OC43/ HKU1), human metapneumovirus (hMPV), adenovirus (AdV), and human bocavirus.

\section{Statistical analysis}

Comparisons of clinical characteristics and clinical outcomes were conducted between patients with $M$. pneumoniae positive and negative group, using an unpaired Student's $t$-test, the Mann-Whitney test, or the Chi-square test (SPSS for Windows 16.0). A p-value $<0.05$ was considered statistically significant. Sensitivity, specificity and predictive values were determined by standard procedures. Clinical diagnostic values of different tests were evaluated by likelihood ratios. Strong evidence to rule in diagnoses in most circumstances when the positive likelihood ratio was above 10 [13].

\section{Results}

During our 2-year prospective study, 125 CAP patients with paired sera meeting the inclusion criteria were enrolled. There were 66 males (52.8\%) and 59 females (47.2\%), and the patients' ages ranged from 14 to 85 years (median 47 years) (see Table 1). Overall, acute $M$. pneumoniae infection was diagnosed in 27/125 (21.6\%) patients showing a fourfold or greater increase in IgG antibody titer of paired sera. Twenty-three patients had causative pathogens other than M. pneumoniae. Among these patients, one was positive for PIV types 1 and 2, and IFVA; one was positive for IFVA and Pseudomonas aeruginosa; one was positive for IFVA and Klebsiella pneumoniae; nine were positive for IFVA; three were positive for adenovirus; three were positive for PIV type 1; one was positive for hMPV; one was positive for HRV; one was positive for PIV type 2; and one was positive for PIV type 3. Patients positive for M. pneumoniae had a median age of 34 years (range 16-77 years), which was significantly younger than $M$. pneumoniae-negative patients (54.5; range 14-85) years, $\mathrm{p}=0.002$ ). M. pneumoniae infections were less likely to have comorbidities ( $\mathrm{p}=0.039)$.

There was no difference in smoking status between the two groups. A similar distribution of most symptoms were found in both groups on admission, with the exception that the duration of fever before admission was longer in the group with $M$. pneumoniae infection than in the group without $(\mathrm{p}=0.013)$. The group with M. pneumoniae infection had a higher mean heart rate $(\mathrm{p}<0.001)$. The two groups had a similar pattern of biochemical and haematological findings. There was no difference in use of antibiotics, treatment status or days after onset of the IgM testing between the two groups.

The results of IgM testing, FQ-PCR and culture were compared (see Table 2). M. pneumoniae positive rates evaluated by IgM antibody testing in acute stage serum (5.6\%), FQ-PCR (17.6\%) and culture (16\%) were lower than the result of paired sera (21.6\%). Among the 27 patients positive for $M$. pneumoniae, only two $(7.4 \%)$ were found to be positive by IgM antibody testing, 11 by FQPCR (40.7\%) and 15 by culture (55.6\%). For all of the 103 non- $M$. pneumoniae infections tested by FQ-PCR, hRNP gene had been successfully amplified (data not shown). Among the discordant results, eight patients showed a fourfold or greater increase in IgG titers, but had negative results in all three methods. Fifteen patients were negative by paired sera, while positive by other methods. Among them, four were positive by both FQ-PCR and culture, one was positive by FQ-PCR and IgM test, one was positive by culture and IgM test, six were positive by FQ-PCR, and three were positive by IgM test. When evaluated by the Japanese Respiratory Society (JRS) scoring system [14], 17 patients positive by paired sera and 40 patients negative by paired sera scored $\geq 4$.

By using paired sera showing a fourfold or greater increase in IgG antibody titer as the "gold standard", diagnostic parameters of the three methods have been evaluated (see Table 3), including sensitivity, specificity, positive and negative predictive values (PPV and NPV respectively) and positive likelihood ratio (PLR). Specificity and NPV of all three methods were high, around 90\% in specificity and around $80 \%$ in NPV. A similarly increasing trend of sensitivity, PPV and PLR was lowest in IgM antibody testing $(7.4 \%, 28.6 \%$ and 1.45 , respectively), intermediate in FQ-PCR $(40.7 \%, 50 \%$ and 3.63 , respectively), and highest in culture (55.6\%, $75 \%$ and 10.9 , respectively).

\section{Discussion}

Our results show that $M$. pneumoniae is an important pathogen of adolescent and adult CAP patients, consistent with the findings of the CAPNETZ report in 2009 [15]. In the present study, we also demonstrate that, compared with non-mycoplasma infections, CAP patients infected with $M$. 
Table 1 Demographic and Clinical features and laboratory findings of studied patients

\begin{tabular}{|c|c|c|c|c|}
\hline & All patients & MP positive & MP negative & $P$ value \\
\hline & $\mathrm{N}=125(\%)$ & $\mathrm{N}=27(\%)$ & $\mathrm{N}=98(\%)$ & \\
\hline Age (years) & $47(14-85)$ & $34(16-77)$ & $54.5(14-85)$ & 0.002 \\
\hline Gender (male) & $66(52.8)$ & $13(48.15)$ & $53(54.08)$ & 0.665 \\
\hline Comorbidities & $30(24.0)$ & $2(7.41)$ & $28(28.57)$ & 0.039 \\
\hline Current smoker & $38(30.4)$ & $6(22.22)$ & $32(32.65)$ & 0.57 \\
\hline \multicolumn{5}{|l|}{ Symptoms } \\
\hline Fever & $110(88.0)$ & $25(92.59)$ & 85 (86.73) & 0.521 \\
\hline Duration from onset (days) & $3(2-5)$ & $4(3-5.5)$ & $3(2-5)$ & 0.013 \\
\hline $\mathrm{T}_{\max }\left({ }^{\circ} \mathrm{C}\right)$ & $39.06 \pm 0.58$ & $39.22 \pm 0.57$ & $39 \pm 0.58$ & 0.113 \\
\hline Cough & $114(91.2)$ & $27(100.00)$ & $87(88.78)$ & 0.119 \\
\hline Sputum production & $83(66.4)$ & $20(74.07)$ & $60(61.22)$ & 0.37 \\
\hline Dyspnea & $22(17.6)$ & $7(25.93)$ & $15(15.31)$ & 0.253 \\
\hline Chest pain & 17 (13.6) & $4(14.81)$ & $13(13.27)$ & 0.761 \\
\hline Digestive symptoms & $11(8.8)$ & $5(18.52)$ & $6(6.12)$ & 0.052 \\
\hline Heart rate/min & $85.36 \pm 18.07$ & $96.7 \pm 16.71$ & $85.16 \pm 11.27$ & 0.000 \\
\hline Respiratory rate/min & $22.32 \pm 12.38$ & $20 \pm 1.78$ & $20.13 \pm 2.86$ & 0.819 \\
\hline \multicolumn{5}{|l|}{ Laboratory findings } \\
\hline White blood cell $\left(10^{9} / \mathrm{L}\right)$ & $8.78 \pm 4.16$ & $8.16 \pm 3.11$ & $8.94 \pm 4.4$ & 0.386 \\
\hline Neutrophil (\%) & $71.73 \pm 12.10$ & $71.11 \pm 9.3$ & $71.89 \pm 12.8$ & 0.77 \\
\hline Lymphocyte (\%) & $19.39 \pm 9.99$ & $18.5 \pm 7.14$ & $19.6 \pm 10.64$ & 0.614 \\
\hline Hematocrit (\%) & $42.15 \pm 28.86$ & $39.86 \pm 4.7$ & $42.7 \pm 32.29$ & 0.659 \\
\hline Hemoglobin $(g / L)$ & $132.54 \pm 20.72$ & $135.68 \pm 16.05$ & $131.71 \pm 21.78$ & 0.387 \\
\hline Platelet $\left(10^{9} / \mathrm{L}\right)$ & $207.77 \pm 68.55$ & $192.77 \pm 50.94$ & $211.76 \pm 72.21$ & 0.211 \\
\hline $\mathrm{ESR}(\mathrm{mm} / \mathrm{hr}) \mathrm{n}=85$ & $41.51 \pm 26.14$ & $40.05 \pm 26.19$ & $41.92 \pm 26.31$ & 0.785 \\
\hline C-reactive protein $(\mathrm{mg} / \mathrm{L}) \mathrm{n}=83$ & $66.78 \pm 65.65$ & $63.75 \pm 51.97$ & $67.74 \pm 69.78$ & 0.841 \\
\hline Antibiotic usage before admission within 1 week & $77(61.6)$ & $18(66.67)$ & $59(60.2)$ & 0.657 \\
\hline Site of care $(n=125)$ & & & & 0.667 \\
\hline Outpatient & $51(40.8)$ & $10(37.04)$ & $41(41.84)$ & \\
\hline Hospitalization & $74(59.2)$ & $17(62.96)$ & $57(58.16)$ & \\
\hline Duration of hospitalization (days) & $14(10-18)$ & $13(6.5-19)$ & $14(11-18)$ & 0.549 \\
\hline Days after onset at the $\lg M$ testing & $4(1-7)$ & $4(2-7)$ & $3.5(1-7)$ & 0.246 \\
\hline
\end{tabular}

Note: The data are presented as means \pm standard deviations, no./total no. (\%), or median (range). Comparison is conducted between MP positive $(n=27)$ and negative $(n=98)$ group.

pneumoniae are younger, less likely to have comorbidities, and have longer duration of fever before admission.

After infection by M. pneumoniae, IgM antibodies appear during the first week of the illness, and reach peak titers during the third week. Martínez et al. have found that the sensitivity of IgM assay was 33.3\% in diagnosis of acute $M$. pneumoniae infection in adult CAP patients [16]. In our study, however, the sensitivity of the IgM assay is only $7.4 \%$ compared with a fourfold increase of IgG titers. In some adult patients, the antibodies are constantly negative or produced 15 days after onset as a result of multiple previous infections $[17,18]$. Therefore, using the IgM assay as the sole test may provide inadequate information.

When compared with PCR, the sensitivity of culture from respiratory specimens has turned out to be as low as $61.5 \%$ [19]. Similarly, She et al. reported that culture was unacceptably insensitive for diagnosing $M$. pneumoniae infection [7]. In our study, however, there was no difference between the positive rates of the two methods, just as Waris et al. found in children [20]. Furthermore, the values of diagnostic parameters, including specificity, sensitivity, positive and negative predictive values and positive likelihood ratio, are the highest among the three methods 
Table $2 M$. pneumoniae detection by IgM antibody testing of acute serum, FQ-PCR, culture and IgG titer of paired sera in adults and adolescents with CAP in Beijing

\begin{tabular}{llccc}
\hline & & \multicolumn{3}{c}{ IgG antibody titers of paired sera with a } \\
fourfold or greater increase (number (\%))
\end{tabular}

evaluated here. In particular, a PLR of 10.9 means that there is a reasonable correlation between culture results and true infection. However, the prolonged turnaround time limits the clinical application of culture.

Compared with serology and culture, PCR (especially real-time PCR) is more rapid, practicable and sensitive, and has been suggested to be more suitable for early diagnosis of acute $M$. pneumoniae infection [21,22]. Touati et al. have evaluated five commercial kits based on PCR, and found that the sensitivities of the kits were 62-98\% [23]. Similarly, Martínez et al. observed the sensitivity, specificity, PPV and NPV of the PCR to be $66.7 \%, 98.5 \%, 78.3 \%$ and $97.3 \%$, respectively [16]. In the present study, however, the sensitivity, specificity, PPV and NPV of the FQ-PCR kit targeted to $16 \mathrm{~S}$ rRNA gene were $40.7 \%, 88.9 \%, 50 \%$ and $84.6 \%$, respectively. The low sensitivity of PCR testing may be due to the low bacterial load resulting from previous antimicrobial treatment or dilution of the sample in the throat swabs below the limit of detection. The detection limit of our FQ-PCR kit is stated as 10 copies per $\mu \mathrm{l}$ in the manual, which is lower than 0.1-1 copies per $\mu$ l that other commercial kits reported [23,24]. In addition, it was found that the efficiency of the PCR assay could be influenced by sample type, and sputum could be superior to other respiratory samples including throat swab [25].

Possible reasons contributing to discordant results between FQ-PCR or culture, and IgG serology have been explored. Four patients (one positive by FQ-PCR and

Table 3 Diagnostic values of laboratory test methods with a fourfold or greater increase of IgG antibody titers of paired sera as the "gold standard"

\begin{tabular}{lccccc}
\hline & Sensitivity (\%) & Specificity (\%) & PPV (\%) & NPV (\%) & PLR \\
\hline IgM result & 7.4 & 94.9 & 28.6 & 78.8 & 1.45 \\
FQ-PCR result & 40.7 & 88.8 & 50 & 84.5 & 3.63 \\
Culture result & 55.6 & 94.9 & 75 & 88.6 & 10.9 \\
\hline
\end{tabular}

PPV: Positive predictive values; NPV: Negative predictive values; PLR: Positive likelihood ratio. culture, and three positive by FQ-PCR) might represent asymptomatic carriage of $M$. pneumoniae as a result of persistence from a previous infection, since these patients' respective CAP etiologies had been identified with IFVA, hMPV, AdV and HRV. Five patients (three positive by FQPCR and culture, and two positive by FQ-PCR or culture and IgM test) showed 2.5-3.8 fold increase of IgG response, which could be explained by an inadequate time interval (mean interval was 17 days). The remaining three patients (only positive by FQ-PCR) were 69, 73 and 74 years old. They failed to develop IgG antibody response in paired sera, which could be interpreted as a deterioration of the immune response due to ageing [26]. These cases highlight the limitations of a serological diagnosis, which could be affected by the timing of specimen collection and the age of the patient.

Recently, in China, Yin et al. demonstrated the good sensitivity and specificity of the JRS scoring system for the early presumptive diagnosis of $M$. pneumoniae pneumonia [14]. In our cohort, we showed that with a fourfold or greater increase of IgG antibody titers of paired sera as the "gold standard", the sensitivity and specificity of JRS scoring were $63 \%$ and $59.2 \%$, respectively. With positivity either by PCR, culture, IgM, or a fourfold increase of IgG as the diagnosis for M. pneumoniae pneumonia, the sensitivity decreased to $61.9 \%$ and specificity increased to $74.7 \%$.

The limitations of the study included: 1) because of cost consideration, we evaluated only one FQ-PCR kit approved by State Food and Drug Administration of China; and 2) in patients who had received antibiotics, including macrolides and fluoroquinolones, the positive rates of culture and FQ-PCR may have been decreased.

\section{Conclusions}

In the defined group of patients, there was a good agreement between positive rate of MP cultivation of throat swabs and acute M. pneumoniae infection (PLR of 10.9). Since the sensitivity was low in all evaluated methods, the logical approach would be to incorporate PCR, culture and serological tests for optimum diagnosis of acute M. pneumoniae infections in adults and adolescents.

\section{Ethical approval}

The study was approved by the Beijing Chao-yang Hospital Ethics Committee and written informed consent was provided by all adults and the parents of patients aged less than 18 years.

\section{Competing interests}

The authors declare that they have no competing interests.

\section{Authors' contributions}

QJX carried out all laboratory tests, performed the statistical analysis, participated in the design and coordination of the study and drafted the manuscript. GL, WJ, DJP, PZH, GY, HM, ZYX and GF participated in enrollment 
of CAP patients and recorded the demographic information. CB and WC participated in the design and coordination of the study, and helped to draft the manuscript. All authors read and approved the final manuscript.

\section{Acknowledgments}

We thank Drs. Liu YM, Li F, Mao Y, Wang CL, Tian GB, Zhao MM, Chen MF, Ding $Y Y$ and Wang $W$, for their help with the collection of specimens and clinical data. We also specially acknowledge the modification of manuscript by Miss Chen SY.

\section{This work was supported by}

Beijing Science and Technology Project (D101100049810002) for Dr. Wang C, New Century Excellent Talents in University (NCET-10-0006) for Dr. Cao B, and Beijing Municipal Health Bureau grant (2011-1004-02) for Dr. Qu JX.

\section{Author details}

${ }^{1}$ Department of Infectious Diseases and Clinical Microbiology, Beijing ChaoYang Hospital, Capital Medical University, Beijing Institute of Respiratory Medicine, Beijing 100020, China. ${ }^{2}$ Beijing Centers for Diseases Control and Prevention, Beijing 100020, China. ${ }^{3}$ Beijing Hai-Dian Hospital, Beijing 100020, China. ${ }^{4}$ YanTai Yu Huang-Ding hospital, Beijing 100020, China. ${ }^{5}$ Peking University People's Hospital, Beijing 100020, China. ${ }^{6}$ The Lu-He teaching hospital of the Capital Medical University, Beijing 100020, China. ${ }^{7}$ Beijing DaXing hospital, Beijing 100020, China. ${ }^{8}$ Wang Jing Hospital of China Academy of Chinese Medical Sciences, Beijing 100020, China. ${ }^{9}$ Beijing Hospital, Ministry of Health, Beijing Institute of Respiratory Medicine, Beijing 100020, China.

Received: 15 October 2012 Accepted: 8 April 2013

Published: 11 April 2013

\section{References}

1. Loens K, Goossens H, leven M: Acute respiratory infection due to mycoplasma pneumoniae: current status of diagnostic methods. Eur J Clin Microbiol Infect Dis 2010, 29(9):1055-1069.

2. Liu Y, Chen M, Zhao T, Wang H, Wang R, Cai B, Cao B, Sun T, Hu Y, Xiu Q, et al: Causative agent distribution and antibiotic therapy assessment among adult patients with community acquired pneumonia in chinese urban population. BMC Infect Dis 2009, 9:31.

3. Cao B, Ren LL, Zhao F, Gonzalez R, Song SF, Bai L, Yin YD, Zhang YY, Liu YM, Guo P, et al: Viral and mycoplasma pneumoniae community-acquired pneumonia and novel clinical outcome evaluation in ambulatory adult patients in China. Eur J Clin Microbiol Infect Dis 2010, 29(11):1443-1448.

4. Lieberman D, Schlaeffer F, Lieberman D, Horowitz S, Horovitz O, Porath A: Mycoplasma pneumoniae community-acquired pneumonia: a review of 101 hospitalized adult patients. Respiration 1996, 63(5):261-266.

5. Principi N, Esposito S, Blasi F, Allegra L: Role of mycoplasma pneumoniae and chlamydia pneumoniae in children with community-acquired lower respiratory tract infections. Clin Infect Dis 2001, 32(9):1281-1289.

6. Waites KB, Talkington DF: Mycoplasma pneumoniae and its role as a human pathogen. Clin Microbiol Rev 2004, 17(4):697-728. table of contents.

7. She RC, Thurber A, Hymas WC, Stevenson J, Langer J, Litwin CM, Petti CA: Limited utility of culture for mycoplasma pneumoniae and chlamydophila pneumoniae for diagnosis of respiratory tract infections. J Clin Microbiol 2010, 48(9):3380-3382.

8. Thacker WL, Talkington DF: Analysis of complement fixation and commercial enzyme immunoassays for detection of antibodies to mycoplasma pneumoniae in human serum. Clin Diagn Lab Immunol 2000, 7(5):778-780.

9. Daxboeck F, Krause $R$, Wenisch C: Laboratory diagnosis of mycoplasma pneumoniae infection. Clin Microbiol Infect 2003, 9(4):263-273.

10. Gnarpe J, Lundback A, Sundelof B, Gnarpe H: Prevalence of mycoplasma pneumoniae in subjectively healthy individuals. Scand J Infect Dis 1992, 24(2):161-164

11. Foy HM: Infections caused by mycoplasma pneumoniae and possible carrier state in different populations of patients. Clin Infect Dis 1993, 17(Suppl 1):S37-S46.

12. Waites KB, Nolte FS: Laboratory diagnosis of mycoplasmal infections. Washington, D.C.: ASM Press; 2001.

13. Deeks JJ, Altman DG: Diagnostic tests 4: likelihood ratios. BMJ Clinical research ed 2004, 329(7458):168-169.
14. Yin YD, Zhao F, Ren LL, Song SF, Liu YM, Zhang JZ, Cao B: Evaluation of the japanese respiratory society guidelines for the identification of mycoplasma pneumoniae pneumonia. Respirology 2012, 17(7):1131-1136.

15. von Baum H, Welte T, Marre R, Suttorp N, Luck C, Ewig S: Mycoplasma pneumoniae pneumonia revisited within the german competence network for community-acquired pneumonia (CAPNETZ). BMC Infect Dis 2009, 9:62.

16. Martinez MA, Ruiz M, Zunino E, Luchsinger V, Avendano LF: Detection of mycoplasma pneumoniae in adult community-acquired pneumonia by PCR and serology. J Med Microbiol 2008, 57(Pt 12):1491-1495.

17. Moule JH, Caul EO, Wreghitt TG: The specific IgM response to mycoplasma pneumoniae infection: interpretation and application to early diagnosis. Epidemiol Infect 1987, 99(3):685-692.

18. Jacobs E: Serological diagnosis of mycoplasma pneumoniae infections: a critical review of current procedures. Clin Infect Dis 1993, 17(Suppl 1):S79-S82.

19. leven M, Ursi D, Van Bever H, Quint W, Niesters HG, Goossens H: Detection of Mycoplasma pneumoniae by two polymerase chain reactions and role of $\mathrm{M}$. Pneumoniae in acute respiratory tract infections in pediatric patients. J Infect Dis 1996, 173(6):1445-1452.

20. Waris ME, Toikka P, Saarinen T, Nikkari S, Meurman O, Vainionpaa R, Mertsola J, Ruuskanen O: Diagnosis of mycoplasma pneumoniae pneumonia in children. J Clin Microbiol 1998, 36(11):3155-3159.

21. Nilsson AC, Bjorkman P, Persson K: Polymerase chain reaction is superior to serology for the diagnosis of acute mycoplasma pneumoniae infection and reveals a high rate of persistent infection. BMC Microbio/ 2008, 8:93.

22. Morozumi M, Hasegawa K, Chiba N, Iwata S, Kawamura N, Kuroki H, Tajima T, Ubukata K: Application of PCR for mycoplasma pneumoniae detection in children with community-acquired pneumonia. J Infect Chemother 2004, 10(5):274-279.

23. Touati A, Benard A, Hassen AB, Bebear CM, Pereyre S: Evaluation of five commercial real-time PCR assays for detection of mycoplasma pneumoniae in respiratory tract specimens. J Clin Microbiol 2009, 47(7):2269-2271.

24. Dumke $R$, Jacobs E: Comparison of commercial and in-house real-time PCR assays used for detection of mycoplasma pneumoniae. J Clin Microbiol 2009, 47(2):441-444.

25. Raty R, Ronkko E, Kleemola M: Sample type is crucial to the diagnosis of mycoplasma pneumoniae pneumonia by PCR. J Med Microbiol 2005, 54(Pt 3):287-291.

26. Dorigo-Zetsma JW, Verkooyen RP, van Helden HP, van der Nat H, van den Bosch JM: Molecular detection of mycoplasma pneumoniae in adults with community-acquired pneumonia requiring hospitalization. J Clin Microbio/ 2001, 39(3):1184-1186.

\section{doi:10.1186/1471-2334-13-172}

Cite this article as: Qu et al:: Accuracy of IgM antibody testing, FQ-PCR and culture in laboratory diagnosis of acute infection by Mycoplasma pneumoniae in adults and adolescents with community-acquired pneumonia. BMC Infectious Diseases 2013 13:172.

\section{Submit your next manuscript to BioMed Central and take full advantage of:}

- Convenient online submission

- Thorough peer review

- No space constraints or color figure charges

- Immediate publication on acceptance

- Inclusion in PubMed, CAS, Scopus and Google Scholar

- Research which is freely available for redistribution 